\title{
8 \\ Conclusions: The Ever-Changing Way Forward
}

\begin{abstract}
In a world that is always changing, 'conclusion' seems an oxymoron. But we can summarize the story. First, that non-stationary data are pervasive in observational disciplines. Second, there are two main sources of non-stationarity deriving from evolutionary change leading to stochastic trends that cumulate past shocks and abrupt changes, especially location shifts, that lead to sudden shifts in distributions. Third, the resulting 'wide sense' non-stationarity not only radically alters empirical modelling approaches, it can have pernicious implications for inter-temporal theory, for forecasting and for policy. Fourth, methods for finding and neutralizing the impacts of distributional shifts from both sources are an essential part of the modeller's toolkit, and we proposed saturation estimation for modelling our changing world.
\end{abstract}

Keywords Theory formulations · Empirical modelling · Forecasting · Policy 
Non-stationarity has important implications for inter-temporal theory, empirical modelling, forecasting and policy. Theory formulations need to account for humans inevitably facing disequilibria, so needing strategies for correcting errors after unanticipated location shifts. Empirical models must check for genuine long-run connections between variables using cointegration techniques, detect past location shifts, and incorporate feedbacks implementing how agents correct their previous mistakes. Forecasts must allow for the uncertainty arising from cumulating shocks, and could switch to robust devices after systematic failures. Tests have been formulated to check for models not being invariant to location shifts, and for policy changes even causing such shifts, potentially revealing that those models should not be used in future policy decisions.

Policy makers must recognise the challenges of implementing policy in non-stationary environments. Regulation of integrated processes, such as atmospheric $\mathrm{CO}_{2}$ concentrations, is challenging due to their accumulation: for example, in climate policy, net-zero emissions are required to stabilise outcomes (see Allen 2015). Invariance of the parameters in policy models to a policy shift is a necessary condition for that policy to be effective and consistent with anticipated outcomes. The possibility of location shifts does not seem to have been included in risk models of financial institutions, even though such shifts will generate many apparently extremely unlikely successive bad draws relative to the prevailing distribution, as seen in Fig. 4.5.

Caution is advisable when acting on forecasts of integrated series or during turbulent times, potentially leading to high forecast uncertainty and systematic forecast failure, as seen in Figs. 7.8 and 7.9. Conversely, as noted in Sect. 3.2, the tools described above for handling shifts in time series enabled Statistics Norway to quickly revise their economic forecasts after Lehmann Brothers' bankruptcy. Demographic projections not only face evolving birth and death rates as in Fig. 2.3, but also sudden shifts, as happens with migration, so like economics, must tackle both forms of non-stationarity simultaneously.

Location shifts that affect the equilibrium means of cointegrating models initially cause systematic forecast failure, then often lead to incorrectly predicting rapid recovery following a fall, but later under-estimating a 
subsequent recovery. Using robust forecasting devices like those recorded in Fig. 7.9 after a shift or forecast failure can help alleviate both problems.

While this book has mainly considered time series data, similar principles apply to cross section and panel observational data. Panel data poses an additional problem of dependence. Time series data has the advantage of historical ordering, enabling sequential factorization to remove temporal dependence. Panel data requires a suitable exogenous ordering to apply sequential factorization which may not be obvious to the modeller. Methods to detect and model outliers and structural breaks may be particularly important in panel data, where individual heterogeneity accounts for much of the data variability. See Pretis et al. (2018) for an example of IIS applied to a fixed-effects panel model looking at the impacts of climate change on economic growth. IIS is equivalent to allowing for a 'fixed effect' for every observation in the panel, and accounting for these country-year individual effects proved invaluable in isolating the effects of climate variation on economic growth.

\section{References}

Allen, M. (2015). Short-lived promise? The science and policy of cumulative and short-lived climate pollutants. Oxford Martin policy paper, Oxford Martin School, Oxford, UK. https://www.oxfordmartin.ox.ac.uk/downloads/ briefings/PolicyNote-SLCPs.pdf.

Pretis, F., Schwarz, M., Tang, K., Haustein, K., and Allen, M. R. (2018). Uncertain impacts on economic growth when stabilizing global temperatures at $1.5^{\circ} \mathrm{C}$ or $2^{\circ} \mathrm{C}$ warming. Philosophical Transactions of the Royal Society, A376, 20160460. https://doi.org/10.1098/rsta.2016.0460. 
Open Access This chapter is licensed under the terms of the Creative Commons Attribution 4.0 International License (http://creativecommons.org/licenses/by/ $4.0 /$ ), which permits use, sharing, adaptation, distribution and reproduction in any medium or format, as long as you give appropriate credit to the original author(s) and the source, provide a link to the Creative Commons license and indicate if changes were made.

The images or other third party material in this chapter are included in the chapter's Creative Commons license, unless indicated otherwise in a credit line to the material. If material is not included in the chapter's Creative Commons license and your intended use is not permitted by statutory regulation or exceeds the permitted use, you will need to obtain permission directly from the copyright holder.

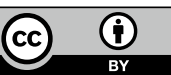

